\title{
Orientation of the cores of hybrid morphology radio sources
}

\author{
M. Cegłowski, M. P. Gawroński, and M. Kunert-Bajraszewska
}

\author{
Toruń Centre for Astronomy, N. Copernicus University, Gagarina 11, 87-100 Toruń, Poland \\ e-mail: magda@astro.uni.torun.pl
}

Received 11 October 2012 / Accepted 12 July 2013

\section{ABSTRACT}

\begin{abstract}
Aims. The FR I/FR II dichotomy is a much debated issue in the astrophysics of extragalactic radio sources. Study of the properties of HYbrid MOrphology Radio Sources (HYMORS) may bring crucial information and lead to a step forward in understanding the origin of FR I/FR II dichotomy. HYMORS are a rare class of double-lobed radio sources where each of the two lobes clearly exhibits a different FR morphology. This article describes follow-up high resolution VLBA observations of the five discovered by us HYMORS. The main aim of the observations was to answer the questions of whether the unusual radio morphology is connected to the orientation of objects towards the observer.

Methods. The milliarcsecond-scale structures are good probes of the galactic medium, the possible asymmetries, and orientation of the central engines of the sources. We obtained the high resolution radio maps of five hybrid radio morphology objects with the VLBA at $C$-band and $L$-band.

Results. The cores of all five sources have been detected at both radio bands. Two of them revealed milliarcsecond core-jet structures, the next two objects showed hints of parsec-scale jets, and the last one remained point-like at both frequencies.

Conclusions. We compared properties of observed milliarcsecond structures of hybrid sources with the larger scale ones previously detected with the VLA. We find that on both scales the fluxes of their central components are similar, which may indicate the lack of additional emission in the proximity of the nucleus. This suggests that jets present on the $\sim 1-10 \mathrm{kpc}$ scale in those objects are FR II-like.

When possible, the detected core-jet structures were used for estimating the core's spatial orientation. The result is that neither the FR I-like nor the FR II-like side is preferred, which may suggest that no specific spatial orientation of HYMORS is required to explain their radio morphology. Their estimated viewing angles indicate they are unbeamed objects. The 178/151 MHz luminosity of observed HYMORS exceed the traditional FR I/FR II break luminosity, indicating they have radio powers similar to FR IIs.
\end{abstract}

Key words. galaxies: active - galaxies: jets - galaxies: nuclei

\section{Introduction}

According to Fanaroff \& Riley (1974), there are two distinct morphological classes of radio galaxies, based on their extended radio emission: Fanaroff-Riley type I and type II sources (FR Is and FR IIs). FR IIs are quite homogeneous in morphology, and their main characteristic is the prominent hotspots at the outer edges of radio lobes. FR I sources show much more diversity (Parma et al. 2002). About half of them have doublelobed morphology similar to FR IIs, and the rest is a mixing of narrow-angle or wide-angle tailed sources (NATs and WATs). The characteristic feature of FR I sources is a lack of hotspots at the outer parts of the radio structure. Fanaroff \& Riley (1974) also introduced the radio luminosity criterion $L_{178} \mathrm{MHz} \sim$ $10^{25.5} \mathrm{~W} \mathrm{H}^{-1} \mathrm{sr}^{-1}$ to distinguish objects between FR I class, which falls bellow this threshold, and the FR II class that is placed above. It has been also suggested that those classes undergo different cosmological evolutions (Wall et al. 1980).

Though the cause of morphological FR I/FR II dichotomy is still unclear, it is worth noticing that there are three main hypotheses that focus on this phenomenon. Several authors claim that it is connected with transition of the intrinsically supersonic jet to transonic/subsonic flow that is being decelerated due to entrainment of thermal plasma in the innermost region of the host galaxy (e.g: Komissarov 1994; Bowman et al. 1996; Bicknell 1995; Kaiser \& Alexander 1997). A second hypothesis links aberration in morphology with a more profound issue of the central engine (Meier et al. 1997; Zirbel \& Baum 1995) or the jet itself (Reynolds et al. 1996; Ghisellini \& Celotti 2001). Last but not least, the scheme attributes FR I/FR II dichotomy to the intergalactic medium, which together with jet power shapes the object morphology (Gopal-Krishna 1991; Gopal-Krishna et al. 1996). Combining those two properties may explain how the hotspot became relatively subsonic to ambient medium, which leads to disruption of the collimated jet due to its weakened Mach disk. Gopal-Krishna \& Wiita (2000, hereafter GKW00) show that there is a rare group of objects $(<1 \%$ of extended FIRST objects; Gawroński et al. 2006) that indeed exhibits two different morphological classes of the jets, and they named them HYMORS (HYbrid MOrphology Radio Source). They point out that the HYMORS class may be essential for understanding the FR I/FR II dichotomy. GKW00 explains that the existence of those objects speaks in favor of a hypothesis in which the medium and jet power play a dominant role in shaping radio source.

Gawroński et al. (2006, hereafter G06) nearly doubled the number of known HYMORS sources. Results presented in G06 strongly support the findings of GKW00, namely that there are two different kinds of jets in HYMORS. Therefore, the existence of a FR-dichotomy as a whole is difficult to reconcile with the explanations that posit only fundamental differences in the central engine. It instead seems that in the case of HYMORS one-sided jet disruption caused by propagation into a large-scale over-density of cold gas is present and that it has a significant 
Table 1. Basic parameters of observed HYMORS.

\begin{tabular}{|c|c|c|c|c|c|c|c|c|c|c|}
\hline $\begin{array}{c}\text { Source name } \\
\text { (1) }\end{array}$ & $\begin{array}{c}\text { RA } \\
\text { h m s } \\
(2)\end{array}$ & $\begin{array}{l}\text { Dec } \\
\circ, 1, \\
(3)\end{array}$ & $\begin{array}{l}z \\
(4)\end{array}$ & $\begin{array}{c}S_{1.4 \mathrm{GHz}} \\
\mathrm{mJy} \\
(5)\end{array}$ & $\begin{array}{l}S_{4.9 \mathrm{GHz}} \\
\mathrm{mJy} \\
(6)\end{array}$ & $\begin{array}{c}\alpha_{4.9 \mathrm{GHz}}^{1.4 \mathrm{GHz}} \\
\text { (7) }\end{array}$ & $\begin{array}{c}S_{1.4 \mathrm{GHz}}^{c} \\
\mathrm{mJy} \\
(8)\end{array}$ & $\begin{array}{c}S_{4.9 \mathrm{GHz}}^{c} \\
\mathrm{mJy} \\
(9)\end{array}$ & $\begin{array}{c}\alpha_{4.9 \mathrm{GHz}}^{1.4 \mathrm{GHz}} \\
(10)\end{array}$ & $\begin{array}{c}L_{178 \mathrm{MHz}} \\
{[\mathrm{W} / \mathrm{Hz}]} \\
(11)\end{array}$ \\
\hline $\mathrm{J} 1154+513$ & 115346.43 & +511704.1 & $0.31^{*}$ & 33.4 & 24.7 & 0.24 & 33.4 & 27,4 & 0.16 & 27.03 \\
\hline $\mathrm{J} 1206+503$ & 120622.39 & +501744.3 & - & 4.5 & 5.8 & -0.20 & 4.5 & 5,8 & -0.21 & - \\
\hline $\mathrm{J} 1313+507$ & 131325.78 & +504206.2 & $0.56^{*}$ & 7 & 6.1 & 0.11 & 7.0 & 6.1 & 0.11 & $27.28^{+}$ \\
\hline $\mathrm{J} 1315+516$ & 131438.12 & +513413.4 & $0.63^{*}$ & 16 & 13.1 & 0.16 & 16 & 13 & 0.16 & $27.10^{+}$ \\
\hline $\mathrm{J} 1348+286$ & 134751.58 & +283629.6 & 0.74 & 4.7 & - & - & - & 44.7 & - & $27.59^{+}$ \\
\hline
\end{tabular}

Notes. Description of the columns: (1) source name; (2) source right ascension (J2000) extracted from FIRST; (3) source declination (J2000) extracted from FIRST; (4) redshift, photometric redshift taken from SDSS (Sloan Digital Sky Survey, York et al. 2000), marked as (*); (5) total flux density at $1.4 \mathrm{GHz}$ taken from FIRST; (6) total flux density at $4.9 \mathrm{GHz}$ taken from GB6; (7) spectral index between 1.4 and $4.9 \mathrm{GHz}$ calculated using flux densities in Cols. (5) and (6) $\left(S \propto v^{-\alpha}\right)$; (8) core flux density measured based on 1.4 GHz VLA observations described in G06; (9) core flux density measured based on $4.9 \mathrm{GHz}$ VLA observations described in G06; (10) core spectral index between 1.4 and $4.9 \mathrm{GHz}$ calculated using flux densities in Cols. (8) and (9) $\left(S \propto v^{-\alpha}\right)$; (11) logarithm of the luminosity at $178 \mathrm{MHz}$, or at $151 \mathrm{MHz}$ if stated differently (+), calculated using the analytical luminosity distance approximation (Wickramasinghe \& Ukwatta 2010).

impact on FR-dichotomy. A number of Chandra observations of HYMORS support this scenario (see e.g. Miller et al. 2006; Miller \& Brandt 2009; Massaro et al. 2009).

Here we describe follow-up observations of the cores of the hybrid sources discovered by G06. High resolution $C$-band and $L$-band observations were made using VLBA in order to determine the orientation of their central engines and compare it with the large-scale radio structures of those sources visible on the VLA maps.

This paper is organized as follow: Sect. 2 describes data reduction and observations, Sect. 3 refers to information concerning individual sources, Sect. 4 presents the results, Sect. 5 contains discussion, and finally Sect. 6 summarizes the main conclusion of this work.

Throughout the paper, we assume the cosmology with $H_{0}=$ $71 \mathrm{~km} \mathrm{~s}^{-1} \mathrm{Mpc}^{-1}, \Omega_{\mathrm{M}}=0.27, \Omega_{\Lambda}=0.73$.

\section{Observations and data reduction}

In our previous work (G06), five new HYMORS were discovered using the VLA Faint Images of the Radio Sky at Twentycentimeters (FIRST) survey (White et al. 1997). Additional $4.9 \mathrm{GHz}$ VLA observations of the selected sources have confirmed that indeed these are large-scale hybrids, with FR I and FR II jet properties. The basic parameters of five target sources have been gathered in Table 1 .

The next step in our study was to look more close-by at the cores of those uncommon sources and compare the orientation of their large-scale features visible on the VLA maps with respect to the identified cores.

We made standard continuum observations of five HYMORS discovered by $\mathrm{G06}$ at the $1.7 \mathrm{GHz}$ and $5 \mathrm{GHz}$ using VLBA (26 and 27 May 2007). Each target source, together with its associated phase reference source, was observed for $\sim 3 \mathrm{~h}$ per frequency in phase-referencing mode of observations. Switching cycles of $8 \mathrm{~min}$ for $L$-band and $5 \mathrm{~min}$ for $C$-band were used. The whole data reduction process was carried out using standard NRAO AIPS procedures. IMAGR was used to produce the final total intensity images (Figs. 1-3). The flux densities of the main components of the target sources were then measured by fitting Gaussian models, using AIPS task JMFIT. The properties of detected components are given in Table 2 . If a given feature is identified at both radio bands the spectral indexes are also calculated.
Table 2. Flux densities of the principal components of the sources measured based on the $1.7 \mathrm{GHz}$ and $5 \mathrm{GHz}$ VLBA observations presented in this paper $\left(S \propto v^{-\alpha}\right)$.

\begin{tabular}{ccccc}
\hline \hline $\begin{array}{c}\text { Source } \\
\text { name } \\
(1)\end{array}$ & $\begin{array}{c}\text { Compo- } \\
\text { nents } \\
(2)\end{array}$ & $\begin{array}{c}S_{1.7 \mathrm{GHz}} \\
\text { mJy } \\
(3)\end{array}$ & $\begin{array}{c}S_{5 \mathrm{GHz}} \\
\mathrm{mJy} \\
(4)\end{array}$ & $\begin{array}{c}\alpha_{5 \mathrm{GHz}}^{1.7 \mathrm{GHz}} \\
(5)\end{array}$ \\
\hline $\mathrm{J} 1154+513$ & $\mathrm{C}$ & $7.5 \pm 0.1$ & $7.6 \pm 0.1$ & -0.01 \\
& $\mathrm{C} 1$ & $2.9 \pm 0.1$ & - & - \\
& $\mathrm{C} 2$ & $0.5 \pm 0.1$ & - & - \\
& $\mathrm{C} 3$ & - & $3.4 \pm 0.1$ & - \\
& $\mathrm{C} 4$ & - & $0.4 \pm 0.1$ & - \\
& $\mathrm{C} 5$ & - & $0.4 \pm 0.1$ & - \\
& $\mathrm{C} 6$ & - & $0.4 \pm 0.1$ & - \\
\hline $\mathrm{J} 1206+503$ & $\mathrm{C}$ & $3.4 \pm 0.1$ & $3.4 \pm 0.1$ & 0.00 \\
\hline $\mathrm{J} 1313+507$ & $\mathrm{C}$ & $5.3 \pm 0.1$ & $3.5 \pm 0.1$ & 0.38 \\
& $\mathrm{C} 1$ & - & $1.3 \pm 0.1$ & - \\
\hline $\mathrm{J} 1315+516$ & $\mathrm{C}$ & $7.2 \pm 0.1$ & $6.2 \pm 0.1$ & 0.14 \\
& $\mathrm{C} 1$ & $0.3 \pm 0.1$ & - & - \\
\hline $\mathrm{J} 1348+286$ & $\mathrm{C}$ & $17.7 \pm 0.4$ & $24.4 \pm 0.1$ & -0.29 \\
& $\mathrm{C} 1$ & $2.4 \pm 0.1$ & $0.7 \pm 0.1$ & 1.12 \\
& $\mathrm{C} 2$ & $1.3 \pm 0.1$ & $0.5 \pm 0.1$ & 0.87 \\
& $\mathrm{C} 3$ & $0.7 \pm 0.1$ & - & - \\
& $\mathrm{C} 4$ & - & $7.3 \pm 0.1$ & - \\
& $\mathrm{C} 5$ & - & $0.5 \pm 0.1$ & - \\
& $\mathrm{C} 6$ & - & $0.3 \pm 0.1$ & - \\
& $\mathrm{C} 7$ & - & $0.3 \pm 0.1$ & - \\
\hline
\end{tabular}

\section{Notes on individual sources}

$J 1154+513$ (Fig. 1) Both $1.7 \mathrm{GHz}$ and $5 \mathrm{GHz}$ VLBA images show core-jet structures pointed towards FR I-like large scale jet. Component $\mathrm{C}$ with a spectral index of $\alpha=-0.01$ visible on both maps is a radio core. The components $\mathrm{C} 1-\mathrm{C} 6$ are parts of the radio jet.

$J 1206+503$ (Fig. 1) A single component is visible in both $1.7 \mathrm{GHz}$ and $5 \mathrm{GHz}$ VLBA images. Flat spectral index $\alpha=0.0$ indicates it is a radio core.

$J 1313+507$ (Fig. 2) The $5 \mathrm{GHz}$ image shows flat spectrum core $\mathrm{C}$ and an additional feature, probably a jet $\mathrm{C} 1$ directed towards FR II-like part of source. The core-jet structure is unresolved in $1.7 \mathrm{GHz}$ observations.

$J 1315+516$ (Fig. 2) The $1.7 \mathrm{GHz}$ image revealed the probable presence of weak jet $\mathrm{C} 1$ directed towards the south; however, the lack of $\mathrm{C} 1$ detection at $\mathrm{C}$-band may indicate that this feature has 

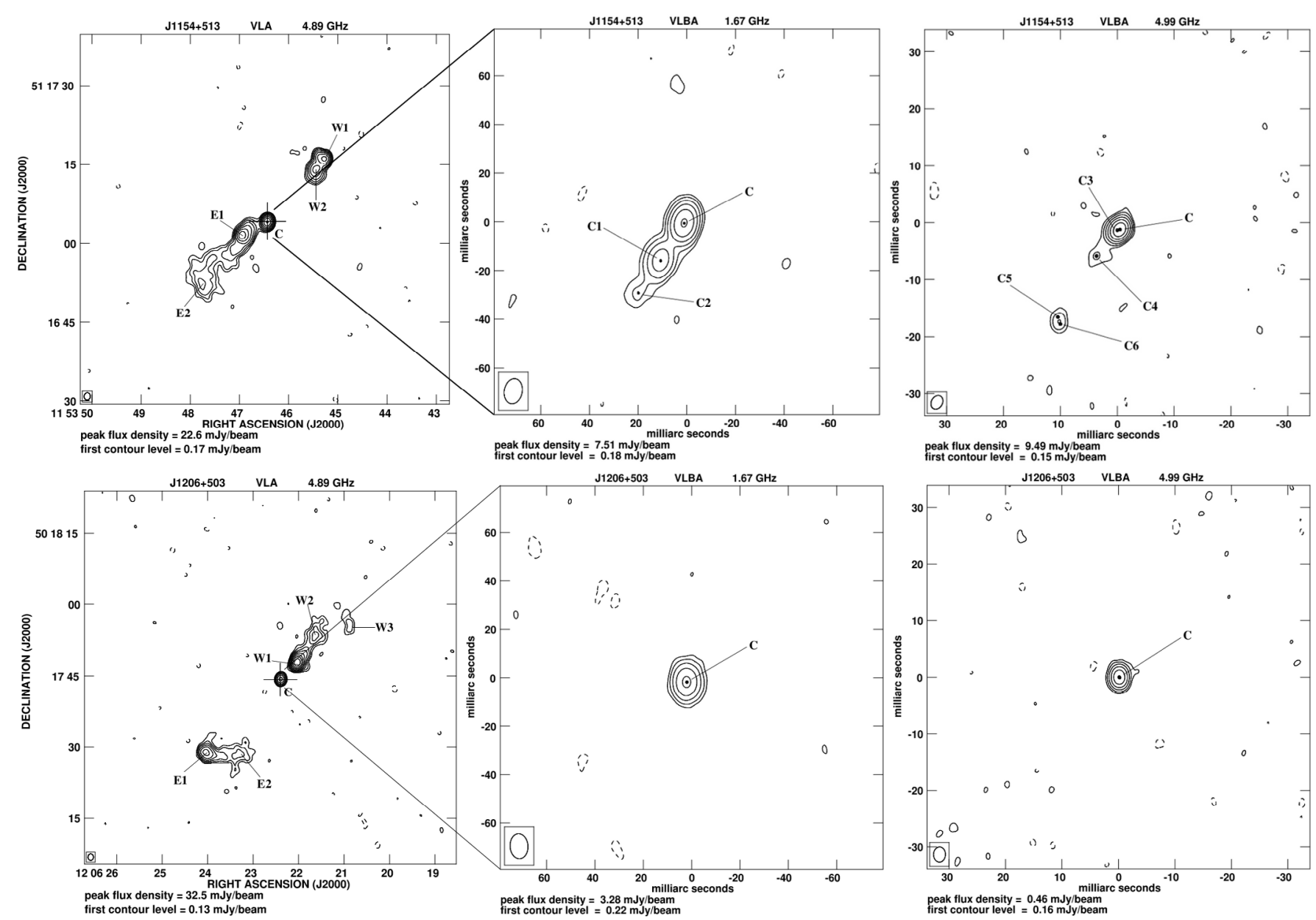

Fig. 1. VLA $C$-band images reprinted from G06 (left) and VLBA $L$-band and $C$-band images (middle and right) of J1154+513 and J1206+503. Contours increase by a factor 2 , and the first contour level corresponds to $\approx 3 \sigma$. A cross indicates the position of an optical object found using the latest version of the SDSS.

a very steep spectrum. The component $\mathrm{C}$ visible in both images is a radio core.

$J 1348+286$ (Fig. 3) Both VLBA images show the presence of a radio jet pointed towards an FR II-like part of the source. The structure of the jet is complicated, and in the $5 \mathrm{GHz}$ image, seven components could be distinguished. The flat spectrum core $\mathrm{C}$ has inverted spectral index $\alpha=-0.29$. This source is classified as a quasar with a measured spectroscopic redshift of $z=0.74$ (Muñoz et al. 2003).

\section{Results}

\subsection{Radio structures}

Here we present higher resolution VLBA observations of five hybrid objects discovered by G06. Two of them revealed a core-jet structure (J1154+513 and J1348+286), and the other two show the probable presence of a weak jet $(\mathrm{J} 1313+507$ and $\mathrm{J} 1315+516$ ). For J1206+503 we have only detected a point-like central component. We analyzed the flux densities of the central components of all five sources measured on VLA and VLBA images (Tables 1 and 2). The summarized flux densities on VLBA scales of four hybrid sources $(\mathrm{J} 1206+503$, J1313+507, $\mathrm{J} 1315+516$, and $\mathrm{J} 1348+286$ ) are comparable to those measured on VLA maps, although for J1348+286 the slightly resolved structure suggests the presence of weak radio jet. The core flux density of J1154+513 measured on the VLA scale is higher than measured on VLBA images, and yet in this case the core has been clearly resolved into a core-jet structure. We conclude that there is no additional diffuse radio emission in the proximity of the nucleus of these sources on $\sim 1-10 \mathrm{kpc}$ scale, which could be anticipated in the case of FR I radio galaxies. We suggest that the potentially present - radio jets of the HYMORS must be very weak on parsec scales, similar to those observed in FR II radio galaxies.

We have gathered the basic properties of the new hybrid objects in Table 1. The spectroscopic or photometric redshift is known for four out of five sources. Their estimated luminosity exceeds the traditional FR I/FR II break luminosity, which at $178 \mathrm{MHz}$ is $\sim 10^{25.5} \mathrm{~W} \mathrm{~Hz}^{-1} \mathrm{sr}^{-1}$ (Fanaroff \& Riley 1974), this indicates that they have radio power similar to FR IIs.

\subsection{Orientation}

In our analysis of the observed HYMORS, we focused on determining of the spatial orientation of the sources and compared the arcsecond-scale resolution VLA images (G06) with the milliarcsecond-scale structures detected during the VLBA observations. Since the radio morphology (the jet and counterjet visibility) can give us a rough estimation of the orientation, we wanted to check that the unusual hybrid morphology is connected with some specific orientation towards the observer. Any clean-cut trend in the orientation could support a possible 

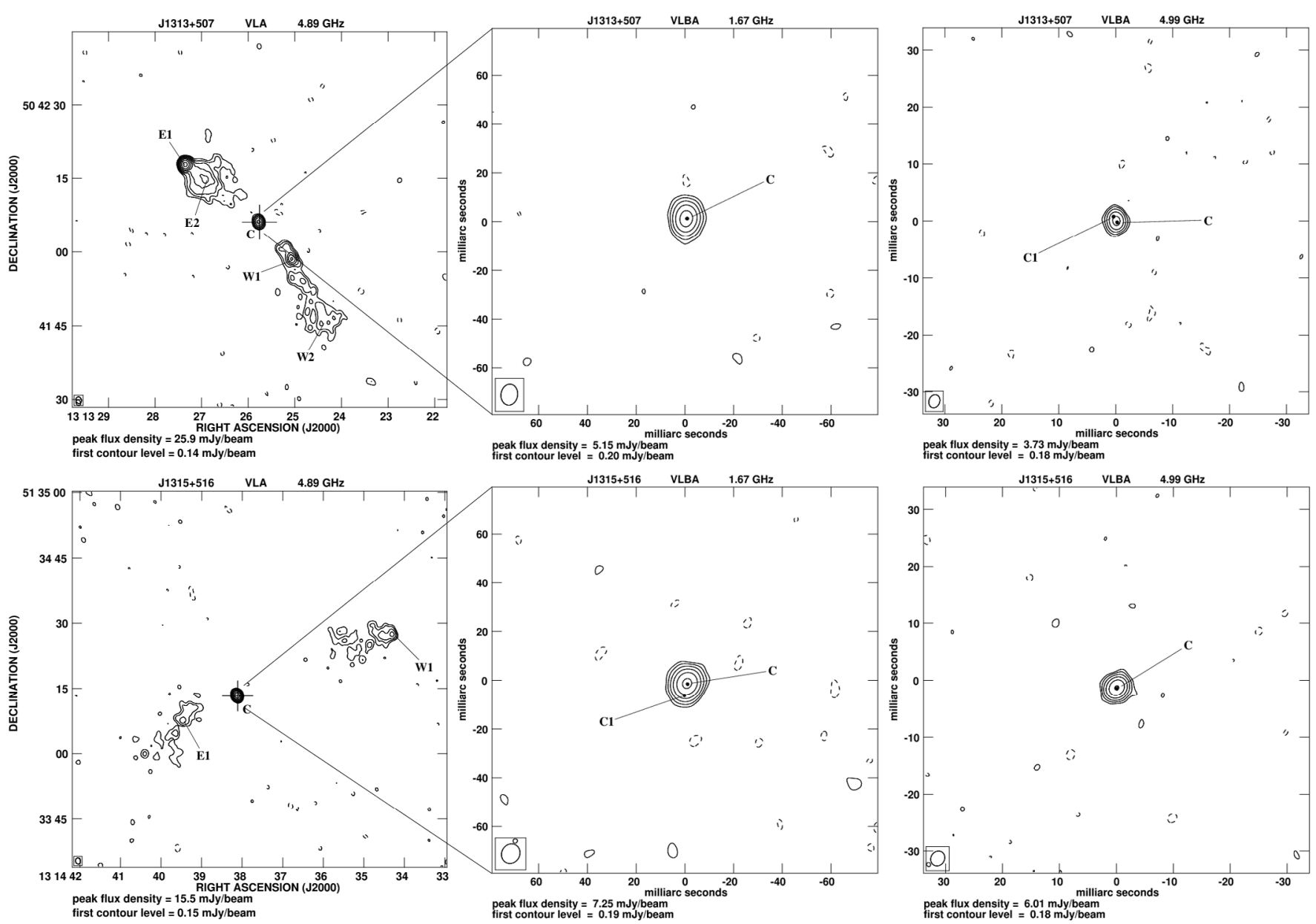

Fig. 2. VLA $C$-band images reprinted from G06 (left) and VLBA $L$-band and $C$-band images (middle and right) of J1313+507 and J1315+516. Contours increase by a factor 2 , and the first contour level corresponds to $\approx 3 \sigma$. A cross indicates the position of an optical object found using the most actual version of the SDSS.

geometric explanation of the HYMORS phenomenon similar to the one presented in Marecki (2012a,b) for sources J1211+743, $3 \mathrm{C} 249.1$, and $3 \mathrm{C} 334$. In the case of $\mathrm{J} 1154+513$ detected jet is clearly directed towards an FR I-like part of the source (Fig. 1). In the case of $\mathrm{J} 1348+286$, the radio structure on VLBA images suggests that the jet is pointing towards the FR II-like side. Also in the case of suspected jets $(\mathrm{J} 1313+507$ and $\mathrm{J} 1315+516)$, none of large-scale side is preferred.

The counterjet is not visible in any of the observed objects, which prevents us from calculating the beaming from a known jet to counterjet brightness ratio. However, to do this we estimated the Doppler factor using the method suggested by Giovannini et al. (2001), which is based on the correlation between the core and total radio power in radio galaxies:

$\log P_{c i 5}=0.62 \log P_{\mathrm{t}}+8.41$,

where $P_{\text {ci5 }}$ is the intrinsic core $5 \mathrm{GHz}$ radio luminosity derived assuming $\gamma=5$, and $P_{\mathrm{t}}$ is the total radio luminosity at $408 \mathrm{MHz}$. The Doppler factor is then estimated by using the $P_{\text {ci5 }}$ and observed $5 \mathrm{GHz}$ radio core luminosity $P_{\mathrm{c}, \mathrm{o}}$ according to the equation;

$P_{\mathrm{c}, \mathrm{o}}=P_{\mathrm{ci} 5} \delta^{2+\alpha}$

assuming spectral index $\alpha=0$. The observed $5 \mathrm{GHz}$ radio core luminosity was calculated using VLA flux from G06 and with a K-correction for $\alpha=0$. The $408 \mathrm{MHz}$ flux were taken from the literature $(\mathrm{J} 1348+286)$ or from an interpolation between $178 / 151 \mathrm{MHz}$ and $1.4 \mathrm{GHz}$ fluxes in other cases. The derived Doppler factor is less than 2.3 for all sources implying viewing angle more than $21^{\circ}$ (for $\gamma=1.5$ ), which can easily prevent us from detecting the counter-jet on the parsec scale.

The above results indicate low beaming in the five studied HYMORS and do not explain the mixture of FR I and FR II morphology. Moreover, the presented VLBA observations do not show that there is any preferable spatial orientation in hybrid objects.

Candidate HYMORS in G06 were selected at at $1.4 \mathrm{GHz}$, a frequency at which Doppler boosting operates. The initial sample constructed from FIRST survey consisted of more than 1700 sources selected in five subareas $\left(F_{1.4 \mathrm{GHz}} \geq 20 \mathrm{mJy}\right.$ and angular size $\theta>8^{\prime \prime}$ ). It is expected that the population of beamed objects is more numerous in the sample constructed at $1.4 \mathrm{GHz}$ than in the case of the similar sample chosen at lower frequencies.

However, if we assume randomly oriented radio sources (e.g. Hough et al. 1992; Barthel 1989), then the number of objects with Doppler factor greater than 1.5 in an unbiased sample decreases from $10 \%$ to $8 \%$ (for $\gamma=1.5,5$ respectively). Therefore it is not surprising that there is only one object in our sample with a Doppler factor greater than 2. The Doppler factor for quasar $\mathrm{J} 1348+286$ amounts to 2.2 . 

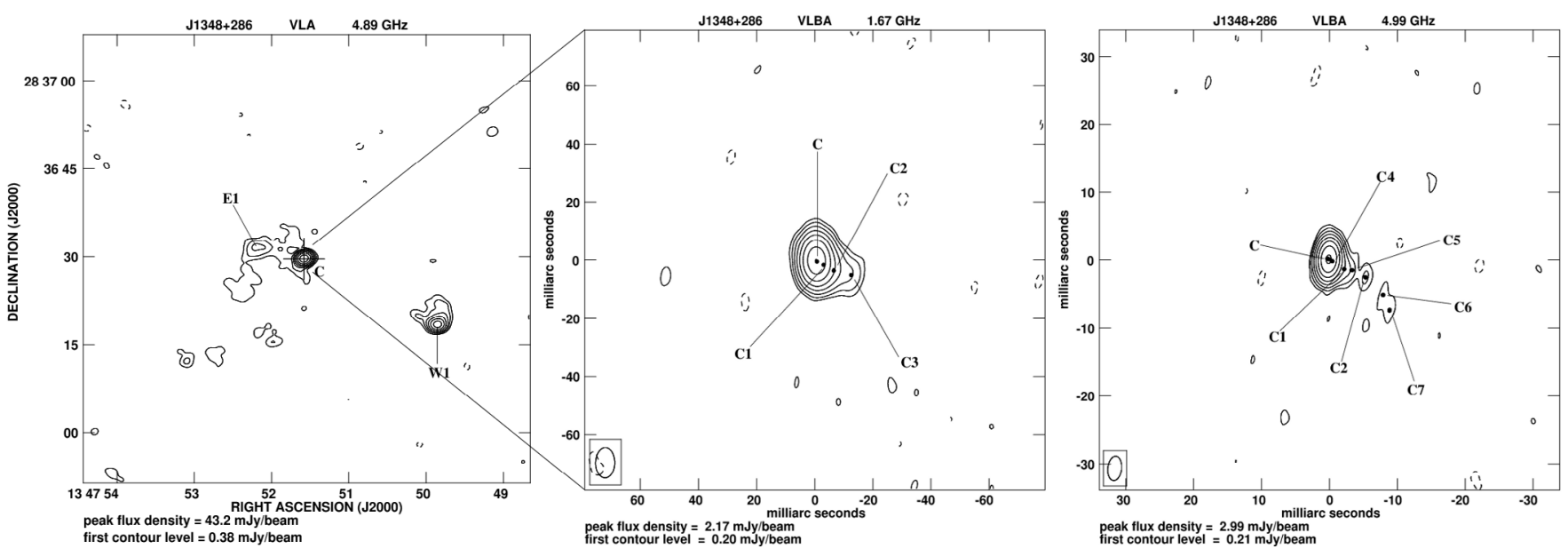

Fig. 3. VLA $C$-band images reprinted from G06 (left) and VLBA $L$-band and $C$-band images (middle and right) of J1348+286. Contours increase by a factor 2 , and the first contour level corresponds to $\approx 3 \sigma$. A cross indicates the position of an optical object found using the most actual version of the SDSS.

\section{Discussion}

The radio observations of HYMORS show that the sources possess an FR I radio morphology on one side of the core and an FR II type structure on the other side (GKW00). In the case of FR II sources the jets are weak but remain relativistic on all scales and terminate abruptly at the end of the jet forming a hotspot. A characteristic feature of FR I sources is the presence of prominent twin jets that passing through a so-called flaring point inflate turbulent lobes with plumes at the end and with no evidence of the strong shocks and hotspots. The flaring point probably marks a transition in the jet where the flow decollimates and starts to decelerate (Laing et al. 1999; Laing \& Bridle 2002). It also corresponds to a sudden increase in the rest-frame emissivity. The length of the deceleration region depends on the total power of the source, and ranges from $\sim 2 \mathrm{kpc}$ for luminosities $<10^{24} \mathrm{~W} \mathrm{~Hz}^{-1}$ at $1.4 \mathrm{GHz}$ to $\sim 10 \mathrm{kpc}$ for the stronger sources. The flaring points have also been observed in WAT objects (Hardcastle \& Sakelliou 2004) and are described as hotspot-like features similar to those visible in FR IIs.

We find such hotspot-like components in the arcsecondscale radio morphologies of HYMORS. These compact, bright features are visible in the inner part of the fuzzy FR I side in all cases (components W1 in the case of J1206+503 and $\mathrm{J} 1313+507$, E1 in $\mathrm{J} 1154+513$, $\mathrm{J} 1315+516$, and $\mathrm{J} 1348+286$, Figs. 1-3 in this paper). They lie closer to the radio core than the hotspots from the FR II side and possess steep spectra. Both the FR I and FR II parts of the hybrid objects show bends and disruption of the radio structure similar to those observed in the WAT objects (Hardcastle \& Sakelliou 2004). We speculate that the bright compact features visible on the FR I side in our hybrid objects could be regions of transition to FR I morphology, which originally started as FR IIs. Such a scenario is considered in many numerical simulations that try to explain the development of the FR I and FR II morphology. In the model of Meliani et al. (2008) a powerful FR II-type jet can be decelerated by a high density nonhomogeneous intergalactic medium and transform to FR I-type on one side and remains FR II on the other side. According to Perucho et al. (2012), the FR II type jet can be disrupted by the growth of helical instability. Indeed our high resolution VLBA observations of HYMORS presented in this paper show similar parsec-scale weak jets on both sides of the radio core.
We looked through the literature for more information about the milliarcsecond observations of hybrid objects. The discussion about the HYMORS was started by GKW00. Since then several articles have appeared containing information about random discovery of possible hybrid sources. Nevertheless, there are very few articles dedicated precisely to HYMORS. Therefore, we focused our investigation on the primary sample of HYMORS defined by the GKW00 and the one described in this paper. GKW00 made a search through the literature and classified six sources as HYMORS. Four out of six objects from their sample have higher resolution VLBI observations, and they possess core-jet structures with the jet pointing towards the FR I side (Pérez-Torres et al. 2004; Beasley et al. 2002; Giroletti et al. 2004) or two-sided jets (Lloyd \& Jones 2002). Two of the objects have been classified as BL Lacs with small viewing angles (Wu et al. 2007). In the case of five hybrid objects, classified by G06 and described there, large beaming is excluded, and the detected parsec-scale jets suggest that neither the FR I-like nor the FR II-like side is preferred. Summing up, there is no complete morphological information about any of the previous studied samples of HYMORS, and/or the classification as hybrid objects are uncertain in some cases. That is why we draw conclusions about the orientation and radio structures only of the HYMORS described in this paper. The new sample of hybrid objects presented here has, for the first time, full information about their structure on different scales, luminosity, and beaming estimations.

Very recently the place of the HYMORS in the evolutionary scheme of AGNs has been discussed by Kunert-Bajraszewska et al. (2010). In this picture HYMORS are typical FR II radio sources with disrupted jet structure on one side of the host galaxy. The break-up of an evolutionary path between FR IIs and HYMORS should take place on scales $\sim 10-15 \mathrm{kpc}$, during the compact steep spectrum (CSS) phase of AGN evolution. However, the reason for such behavior is not obvious, and its explanation is connected with the ongoing discussion about the nature of the FR division. The observations of the variety of the large-scale radio morphologies connected with a wide range in AGN radio loudness suggest that the combination of multiple factors is needed to explain the origin of FR I/FR II dichotomy. There is a growing number of publications suggesting that the luminosity threshold $\left(L_{178 \mathrm{MHz}}\right)$ is either insufficient or misleading in terms of FR I/FR II dichotomy. Kharb et al. (2010) 
show examples of sources that exhibit different morphological classes than would be suggested of taking the luminosity criterion into account. Moreover, X-ray and the optical spectroscopic studies (Best \& Heckman 2012; Buttiglione et al. 2010) support the division of radio sources based on line ratios (LEG and high excitation galaxies - HEG types) rather then their observed morphology, and link the discussion to the properties of central engine: black hole spin or mode of accretion. In this classification, weak FR IIs (LEGs) fueled via radiatively inefficient flows at low accretion rates can be grouped together with FR Is (e.g. Rigby et al. 2008; Best \& Heckman 2012). As has recently been shown, the HEG/LEG division is present also among young radio objects: CSS and Gigahertz-Peaked Spectrum (GPS) sources (Kunert-Bajraszewska \& Labiano 2010), which are considered as progenitors of large scale FR I and FR II sources. The above analysis and conclusions concerning the new sample of HYMORS suggest they are FR IIs that, after the interactions with the inhomogeneous medium, changed their morphology. However, the still open question is whether they are FR II HEGs or LEGs. Spectroscopic studies of HYMORS are needed to determine the properties of their central engines, which could appear an important element of the puzzle called HYMORS.

\section{Summary}

We have presented here the first high-resolution VLBA observations of five hybrid sources that were discovered by us in order to determine if the unusual radio morphology is connected with the orientation of objects towards the observer. The sample gives complete, multifrequency information about the radio structure of HYMORS on different scales, luminosity, and beaming estimations. These are unbeamed objects with viewing angles more than $21^{\circ}$ for the inner parsec scale structures. Their VLBA observations do not suggest there is a preferable spatial orientation in hybrid objects. Two of them revealed milliarcsecond core-jet structure and another two probable weak jets. We suggest that very weak parsec-scale radio jets could be present in all objects, which make HYMORS cores similar in properties to FR II radio galaxies. Moreover, the estimated luminosity of observed HYMORS exceeds the traditional FR I/FR II break luminosity indicating they have radio powers similar to FR IIs.

Based on the performed analysis of the sample of new hybrid objects we suggested that HYMORS are FR IIs evolving in a heterogeneous environment. However, this could be not the only factor making HYMORS different. The interesting question is wheter the hybrid objects are FR II HEGs or weaker FR II LEGs that are grouped together with FR Is and seem to be on the evolutionary path with CSS LEGs.

Acknowledgements. We thank an anonymous referee for carefully reading the manuscript and for the constructive comments. The VLA and VLBA is operated by the US National Radio Astronomy Observatory, which is a facility of the National Science Foundation operated under cooperative agreement by Associated Universities, Inc. This research made use of the NASA/IPAC Extragalactic Database (NED), which is operated by the Jet Propulsion Laboratory, California Institute of Technology, under contract with the National Aeronautics and Space Administration. Use has been made of the fourth release of the Sloan Digital Sky Survey (SDSS) Archive. Funding for the creation and distribution of the SDSS Archive was provided by the Alfred P. Sloan Foundation, the Participating Institutions, the National Aeronautics and
Space Administration, the National Science Foundation, the US Department of Energy, the Japanese Monbukagakusho, and the Max Planck Society. The SDSS Web site is http://www.sdss.org/. The SDSS is managed by the Astrophysical Research Consortium (ARC) for the Participating Institutions. The Participating Institutions are The University of Chicago, Fermilab, the Institute for Advanced Study, the Japan Participation Group, The Johns Hopkins University, Los Alamos National Laboratory, the Max-Planck-Institute for Astronomy (MPIA), the Max-Planck-Institute for Astrophysics (MPA), New Mexico State University, University of Pittsburgh, Princeton University, the United States Naval Observatory, and the University of Washington.

\section{References}

Barthel, P. D. 1989, ApJ, 336, 606

Beasley, A. J., Gordon, D., Peck, A. B., et al. 2002, ApJS, 141, 13

Best, P. N., \& Heckman, T. M. 2012, MNRAS, 421, 1569

Bicknell, G. V. 1995, ApJS, 101, 29

Bowman, M., Leahy, J. P., \& Komissarov, S. S. 1996, MNRAS, 279, 899

Buttiglione, S., Capetti, A., Celotti, A., et al. 2010, A\&A, 509, A6

Cawthorne, T. V., Scheuer, P. A. G., Morison, I., \& Muxlow, T. W. B. 1986, MNRAS, 219, 883

Gawroński, M. P., Marecki, A., Kunert-Bajraszewska, M., \& Kus, A. J. 2006, A\&A, 447, 63

Ghisellini, G., \& Celotti, A. 2001, A\&A, 379, L1

Giovannini, G., Cotton, W. D., Feretti, L., Lara, L., \& Venturi, T. 2001, ApJ, 552, 508

Giroletti, M., Giovannini, G., Taylor, G. B., \& Falomo, R. 2004, ApJ, 613, 752

Gopal-Krishna, 1991, A\&A, 248, 415

Gopal-Krishna, \& Wiita, P. J. 2000, A\&A, 363, 507

Gopal-Krishna, Wiita, P. J., \& Hooda, J. S. 1996, A\&A, 316, L13

Fanaroff, B. L., \& Riley, J. M. 1974, MNRAS, 167, 31

Hardcastle, M. J., \& Sakelliou, I. 2004, MNRAS, 349, 560

Hough, D. H., Readhead, A. C. S., Wood, D. A., Jr., \& Feldmeier, J. J. 1992, ApJ, 393, 81

Kaiser, C. R., \& Alexander, P. 1997, MNRAS, 286, 215

Kaiser, C. R., \& Best, P. N. 2007, MNRAS, 381, 1548

Kharb, P., Lister, M. L., \& Cooper, N. J. 2010, ApJ, 710, 764

Komissarov, S. S. 1994, MNRAS, 269, 394

Kunert-Bajraszewska, M., \& Labiano, A. 2010, MNRAS, 408, 2279

Kunert-Bajraszewska, M., Gawroński, M. P., Labiano, A., \& Siemiginowska, A., 2010, MNRAS, 408, 2261

Laing, R. A., \& Bridle, A. H. 2002, MNRAS, 336, 1161

Laing, R. A., Parma, P., de Ruiter, H. R., \& Fanti, R. 1999, MNRAS, 306, 513

Laing, R. A., Guidetti, D., Bridle, A. H., Parma, P., \& Bondi, M. 2011, MNRAS, 417, 2789

Lloyd, B. D., \& Jones, P. A. 2002, MNRAS, 331, 717

Marecki, A. 2012a, A\&A, 544, A2

Marecki, A. 2012b, A\&A, 545, A132

Massaro, F., Harris, D. E., Chiaberge, M., et al. 2009, ApJ, 696, 980

Meier, D. L., Edgington, S., Godon, P., Payne, D. G., \& Lind, K. R. 1997, Nature, 388, 350

Meliani, Z., Keppens, R., \& Giacomazzo, B. 2008, A\&A, 491, 321

Miller, B. P., \& Brandt, W. N. 2009, ApJ, 695, 755

Miller, B. P., Brandt, W. N., Gallagher, S. C., et al. 2006, ApJ, 652, 163

Muñoz, J. A., Falco, E. E., Kochanek, C. S., Lehár, J., \& Mediavilla, E. 2003, ApJ, 594, 684

Parma, P., Murgia, M., de Ruiter, H. R., \& Fanti, R. 2002, New Astron. Rev., 46, 313

Pérez-Torres, M. A., Marcaide, J. M., Guirado, J. C., \& Ros, E. 2004, A\&A, 428, 847

Perucho, M., Kovalev, Y. Y., Lobanov, A. P., Hardee, P. E., \& Agudo, I., 2012, ApJ, 749, 55

Reynolds, C. S., Di Matteo, T., Fabian, A. C., Hwang, U., \& Canizares, C. R. 1996, MNRAS, 283, L111

Rigby, E. E., Best, P. N., \& Snellen, I. A. G. 2008, MNRAS, 385, 310

York, D. G., Adelman, J., Anderson, J. E., et al. 2000, ApJ, 120, 1579

Wall, J. V., Pearson, T. J., \& Longair, M. S. 1980, MNRAS, 193, 683

White, R. L., Becker, R. H., Helfand, D. J., \& Gregg, M. D. 1997, ApJ, 475, 479

Wickramasinghe, T., \& Ukwatta T. N. 2010, MNRAS, 406, 548

Wu, Z., Jiang, D. R., Gu, M., \& Liu, Y. 2007, A\&A, 466, 63

Zirbel, E. L., \& Baum, S. A. 1995, ApJ, 448, 521 